\title{
Effects of acceptance of disability on death or dialysis in chronic kidney disease patients: a 3-year prospective cohort study
}

Hsin-Hung Chiang ${ }^{1,2+}{ }^{+}$, Hanoch Livneh ${ }^{3{ }^{\dagger}}$, How-Ran Guo ${ }^{4,5 \dagger}$, Mei-Ling Yen ${ }^{1}$ and Tzung-Yi Tsai ${ }^{4,6,7^{*}}$

\begin{abstract}
Background: Acceptance of disability (AOD) is a useful construct that assesses the ability of a patient to psychologically cope with chronic diseases, but its effect on long-term outcomes of patients with chronic kidney disease (CKD) remains unclear. This study aimed to evaluate the relation between AOD level and clinical outcomes in a cohort of CKD patients in Taiwan.

Methods: 262 CKD patients without dialysis at a hospital in Taiwan were consecutively recruited, from 2010 to 2011, and followed up for 3 years. At enrollment, demographic and clinical data were obtained, including baseline level measurement of AOD, using the Acceptance of Disability Scale-Revised (AODS-R). During follow-up, the authors assessed the effect of AOD on progression to dialysis and all-cause mortality by using Cox proportional hazard regression analysis.
\end{abstract}

Results: Of the patients included in the analyses, 145 (55.3\%) whose total scores of AOD were below the median (86.00) were regarded as having low AOD at enrollment. At the end of 3-year follow-up, 25 have died and 57 initiated dialysis. Participants with low AOD were more likely to have the composite end-point of progression to dialysis or death (adjusted hazard ratios [AHR] $=1.89,95 \%$ confidence interval [CI]: 1.18-3.20). In addition, CKD stage at IV or above and hemoglobin level were found to be associated with the occurrence of the composite end-point.

Conclusion: AOD was associated with an increased risk for poor clinical outcomes, thus suggesting that prompt awareness and management of the psychological reactions may improve clinical outcomes of patients with CKD.

Keywords: Acceptance of disability, Chronic kidney disease, Dialysis, Mortality, Taiwan

\section{Background}

Chronic kidney disease (CKD) has become a global public health burden over the past decades [1]. Due to the irreversible nature of CKD, most patients gradually progress to end-stage renal disease (ESRD) and require dialysis or renal transplantation. The global ESRD prevalence is estimated to continue to rise at an annual rate of $7 \%$ and exceed 2 million by 2010 [2]. In Taiwan, where the prevalence is among the highest in the world,

\footnotetext{
* Correspondence: dm732024@tzuchi.com.tw

${ }^{\dagger}$ Equal contributors

${ }^{4}$ Department of Environmental and Occupational Health, College of Medicine, National Cheng Kung University, Tainan, Taiwan

${ }^{6}$ Department of Medical Research, Dalin Tzuchi Hospital, The Buddhist Tzuchi Medical Foundation, No. 2, Minsheng Rd., Dalin Township, Chiayi County 62247ROC, Taiwan

Full list of author information is available at the end of the article
}

ESRD has put a substantial burden on healthcare resources. A governmental report in 2012 revealed that while Taiwanese ESRD patients constituted less than $0.3 \%$ of the population, they took up about $7 \%$ of the total healthcare budgets, surpassing that of cancer patients [3].

In addition to the enormous economic impact, CKD is also a significant public health burden. A cohort study in Taiwan of 462,293 subjects reported that nearly $10.3 \%$ of deaths were due to CKD, and that $40 \%$ of CKD deaths occurred in patients younger than 65 years-old [4]. Therefore, CKD patients typically experience many symptoms over a prolonged period of time and are frequently diagnosed with psychiatric disorders, such as depression. A meta-analysis showed that depression was common in patients with CKD, with a pooled prevalence 
of $21 \%$, approximately two- to four-fold higher than that in the general population [5]. Furthermore, CKD patients with depressive symptoms have over three-fold increased risk of death relative to those without the symptoms [6], suggesting that the improvement of psychological adjustment would prolong the survival rate.

In general, a psychological intervention program for patients with chronic diseases appears to help them to accept their disabilities, learn to better cope and live with their diseases, and adapt to the ensuing physical and psychosocial sequelae [7]. Acceptance of disability (AOD) is considered a key factor that accounts for this improvement in psychosocial adjustment $[8,9]$. AOD is associated with viewing one's disability as a manageable barrier that does not devalue life or minimize one's remaining abilities, and this can be achieved through personal coping efforts and the realization that one can successfully overcome many of life's restrictions and limitations [10]. To specifically measure AOD, Linkowski developed an AOD scale based on concepts inherent in the notion of acceptance of loss [11]. Numerous studies have used this scale to examine disease and disability acceptance among patients with chronic conditions such as Ehlers-Danlos syndrome [12], diabetes [13], spinal cord injury [8], and stroke [14]. Overall, these results indicated that the higher the level of disability acceptance, the more successful is the coping with the condition and the better is the perceived quality of life of the patients. Nonetheless, the long-term effects of AOD on clinical outcomes in patients with chronic diseases remain unclear. To fill this gap, we conducted a study to examine the relationships between AOD and two clinical outcomes, namely, progression to dialysis and all-cause mortality, among CKD patients in Taiwan.

\section{Methods \\ Study design and population}

We recruited consecutive CKD patients from either outpatient or inpatient departments, located at a hospital in southern Taiwan, from January 2010 to December 2011. The eligibility criteria at baseline included: (1) age of at least 20 years; (2) no physician-based diagnosis of alcohol abuse, dementia, psychosis, or depression; (3) ability to express opinions in Mandarin or Taiwanese; (4) diagnosis of CKD by a physician, but not yet undergoing dialysis; and (5) willingness to consent to a blood test on the survey date. Before enrollment, all participants received detailed written and verbal information regarding the aims and protocol of the study and signed informed consents. To ensure participants' anonymity, all questionnaires were marked with encryption code to facilitate data analysis, but lacking any personal identifiers. This study was approved by the institutional review board of the Dalin Tzuchi Hospital, Buddhist Tzuchi Medical Foundation, Taiwan (No. B10201011).

\section{Measures}

\section{Assessment of AOD level}

AOD was assessed by the revised version of the AOD scale (AODS-R), developed by Groomes and Linkowski [9]. It is a 32-item questionnaire that was developed as a brief multidimensional self-reporting instrument for the assessment of four domains: enlargement of scope of values, subordination of physique, containment of disability effects, and transformation from comparative to asset values. Each item is rated on a 4-point Likert scale, ranging from 1 (strongly disagree) to 4 (strongly agree). Thus, the AODS-R total score ranges from 32 to 128 , where higher scores indicate a higher level of disability acceptance. Ten of the items address positive values. The remaining 22 items address negative values. A principal component analysis extracted four factors corresponding to the psychometric characteristics of the original version, thus providing support for the conceptual validity of the AODS-R [9]. Previous studies have demonstrated that the AODS- $\mathrm{R}$ has good internal consistency in different groups of patients with chronic illnesses and disabilities [14-16]. The data collected in our study yielded a Cronbach's $\alpha$ of 0.92 for the scale.

\section{Covariates}

Patient interviews and reviews of medical records were performed to collect the information on demographic and clinical characteristics at enrollment. The demographic data considered included sex, age, marital status, educational level, job status, living status, religious beliefs, monthly income, and certain lifestyle factors, such as use of alcohol and tobacco, exercise habits, and presence of sleep disturbances. Alcohol consumption was recorded as "less than 2 times per week" or "at least two times per week"; smoking status was recorded as "nonsmoker" or "current or ex-smoker." Those who exercised regularly (that is, weekly) were regarded as having "exercise habits". Sleep disturbance was defined as waking up at night more than twice without external factors during the week before the interview. Clinical characteristics included the presence of a chronic disease (i.e. at least one of the following: diabetes mellitus, hypertension, heart disease, or stroke), CKD stage, hemoglobin level, and serum albumin level.

\section{Primary clinical outcomes}

The primary clinical outcomes comprised death (allcause mortality) and progression to dialysis. The date of initiation of dialysis was determined during monthly face-to-face interviews at outpatient clinics or by telephone. If the researchers suspected that a patient has 
died, they contacted the participant's family directly for confirmation, and to obtain the date of death. All participants were followed from the date of enrollment until death or the end of 3-year follow-up, whichever came first.

\section{Statistical analysis}

After using the median of the range of obtained AOD scores as the cut-off, we split participants into two groups: "high" and "low" AOD levels. The mean, standard deviation (SD), and percentage of demographic and disease data between the two AOD groups were then compared employing two-sample $t$ tests for continuous variables and chi square tests for categorical variables. Next, incidence rates of adverse events were presented as the number of cases per 1000 person-months (PMs). Finally, we applied the Cox proportional hazard model to compute the AHR and its $95 \% \mathrm{CI}$ for primary outcomes associated with a low AOD level. Assumptions of the proportional hazard models were confirmed by using log-log plots and cumulative Schoenfeld residual plots. All inferential analyses were two-tailed using an alpha level of 0.05 , and all statistical analyses were performed using SAS version 9.3 (SAS Institute Inc., Cary, NC, USA).

\section{Results}

\section{Basic characteristics of participants}

Of the 267 consecutive qualified CKD patients, 3 refused to participate in the follow-up survey and 2 were excluded because primary outcomes occurred within the first month of follow-up, leaving insufficient time for the establishment of temporal relationship. Therefore, data on 262 patients were included in the final analysis. The mean age of patients was 64.3 years, and $61.5 \%$ were men (Table 1). The mean AOD score was 85.02 ( \pm 5.36 ), and $55.3 \%(145 / 262)$ of the participants scored at a low AOD level (at AOD score below the median of 86.00).

Patients with a low AOD level were more likely to have a low educational level $(p=0.04)$, did not exercise regularly $(p=0.01)$, had sleep disturbance $(p=0.02)$, were diagnosed with CKD stage IV or above $(p=0.03)$, had concomitant chronic diseases $(p=0.04)$, and had a low hemoglobin level $(p=0.02)$ (Table 1).

\section{Incidence and hazard ratios for death and initiation of dialysis}

Overall, the mean follow-up was 31.17 months, and 82 patients $(31.3 \%)$ either died $(n=25,9.5 \%)$ or progressed to dialysis $(n=57,21.8 \%)$. CKD patients with low AOD were more likely to progress to dialysis $(27.6 \% v s$. $14.5 \% ; p=0.03)$ and die $(13.1 \%$ vs. $5.1 \% ; p=0.01)$. After adjustment for the significant factors in the univariate analysis, we found that lower AOD was associated with an elevated but non-significant risk of death $(\mathrm{AHR}=1.82 ; 95 \% \mathrm{CI}: 0.90-4.90)$. In contrast, AOD level was associated with a statistically significant risk of progression to dialysis $(\mathrm{AHR}=1.95 ; 95 \% \mathrm{CI}$ : 1.04-3.34) (Table 2).

\section{Multivariable analysis of factors associated with the composite end-point}

When we combined death and progression to dialysis as a composite end-point, we found that low AOD score was associated with an increased risk $(\mathrm{AHR}=1.89 ; 95 \%$ CI: 1.18-3.20) after adjustment for potential confounding factors. The other independent risk factors included CKD stage IV or above (AHR $=1.82$; $95 \%$ CI: $1.21-$ $4.21)$, and a low hemoglobin level $(\mathrm{AHR}=0.81 ; 95 \% \mathrm{CI}$ : 0.70-0.92) (Table 3).

\section{Discussion}

The present 3-year longitudinal follow-up study of CKD patients who were not yet on dialysis showed that those with low AOD had an increased risk for progression to dialysis, which is consistent with findings of previous studies that CKD patients who experience negative emotions tend to also experience worse clinical manifestations $[5,17]$. A study by Fischer and colleagues [18] also found that depressive symptoms predicted a $15 \%$ greater risk of progression to ESRD among patients with CKD, but this increased risk did not reach statistical significance. This discrepancy in findings could be partially attributed to difference in potential confounders measured in both analyses. It could also be attributed to different definitions of disease stage between the two studies since Fischer et al. [18] directly used the value of glomerular filtration rate (GFR) to evaluate the disease severity of CKD, and not classification of stage.

There are at least several potential reasons for the association between low AOD and poor clinical outcomes. First, negative emotions, such as depression, are known to be associated with treatment non-adherence [19] and unhealthy lifestyle such as malnutrition and physical inactivity that may accelerate the decline in serum albumin and thus progression of CKD [20, 21]. Systematic inflammation induced by a distressed mood may be another plausible explanation; many studies demonstrated that CKD patients with depressive symptoms had higher levels of circulating inflammatory markers, such as interlukin-6 (IL-6), tumor necrosis factor- $\alpha$ (TNF- $\alpha$ ), and C-reactive protein (CRP), all of which play important roles in the pathogenesis of cardiovascular diseases and worsen the prognosis of CKD [5, 22]. Third, negative mood influences the sensitivity to glucocorticoid hormones and leads to immunosuppression through activation of the hypothalamic-pituitary-adrenal (HPA) axis [23], which substantially increases the risk of acute 
Table 1 Demographic and clinical characteristics of all CKD patients with low and high AOD

\begin{tabular}{|c|c|c|c|c|}
\hline Variables & All subjects $(n=262)$ & Low AOD $(n=145)$ & High AOD $(n=117)$ & $p$ \\
\hline \multicolumn{5}{|c|}{ Demographic characteristics } \\
\hline Age (years) & $64.26 \pm 13.25$ & $64.08 \pm 12.14$ & $65.29 \pm 14.25$ & 0.47 \\
\hline \multicolumn{5}{|l|}{ Gender } \\
\hline Male & $161(61.5)$ & $83(57.2)$ & $78(66.7)$ & \multirow[t]{2}{*}{0.12} \\
\hline Female & $101(38.5)$ & $62(42.8)$ & 39 (33.3) & \\
\hline \multicolumn{5}{|l|}{ Educational level } \\
\hline $\operatorname{High}\left(\geq 12^{\text {th }}\right.$ grade $)$ & $66(25.2)$ & $45(31.0)$ & $51(43.6)$ & \multirow[t]{2}{*}{$0.04^{*}$} \\
\hline $\operatorname{Low}\left(<12^{\text {th }}\right.$ grade $)$ & $196(74.8)$ & $100(69.0)$ & 66 (65.4) & \\
\hline \multicolumn{5}{|l|}{ Marital status } \\
\hline Single & $18(6.9)$ & $12(8.3)$ & $6(5.1)$ & \multirow[t]{2}{*}{0.32} \\
\hline Married & $244(93.1)$ & $133(91.7)$ & $111(94.9)$ & \\
\hline \multicolumn{5}{|l|}{ Job status } \\
\hline Employed & $73(27.9)$ & $43(29.7)$ & $30(25.6)$ & \multirow[t]{2}{*}{0.47} \\
\hline Unemployed & $189(72.1)$ & $102(70.3)$ & $87(74.4)$ & \\
\hline \multicolumn{5}{|l|}{ Living status } \\
\hline Alone & $22(8.4)$ & $12(8.3)$ & $10(8.6)$ & \multirow[t]{2}{*}{0.92} \\
\hline Cohabitating & $239(91.6)$ & $133(91.7)$ & $106(91.4)$ & \\
\hline \multicolumn{5}{|l|}{ Monthly income ${ }^{\star}$} \\
\hline$\geq$ NT\$30,001 & $50(19.1)$ & 27 (18.6) & $23(19.7)$ & \multirow[t]{2}{*}{0.83} \\
\hline$\leq \mathrm{NT} \$ 30,000$ & $212(80.9)$ & $118(81.4)$ & $94(80.3)$ & \\
\hline \multicolumn{5}{|l|}{ Religious beliefs } \\
\hline Yes & $223(85.1)$ & $123(84.8)$ & $100(85.5)$ & \multirow[t]{2}{*}{0.88} \\
\hline No & $39(14.9)$ & $22(15.2)$ & $17(14.5)$ & \\
\hline \multicolumn{5}{|l|}{ Alcohol drinking } \\
\hline Low ( $\leq 1$ times/week) & $247(94.3)$ & $136(93.8)$ & $111(94.9)$ & \multirow[t]{2}{*}{0.71} \\
\hline High ( $\geq 2$ times/week) & $15(5.7)$ & $9(6.2)$ & $6(5.1)$ & \\
\hline \multicolumn{5}{|l|}{ Cigarette smoking } \\
\hline Yes & $107(40.8)$ & $59(40.7)$ & $48(41.0)$ & \multirow[t]{2}{*}{0.95} \\
\hline No & $155(59.2)$ & $86(59.3)$ & $69(59.9)$ & \\
\hline \multicolumn{5}{|l|}{ Regular exercise } \\
\hline Yes & $171(65.3)$ & 85 (58.6) & $86(73.5)$ & \multirow[t]{2}{*}{$0.01^{*}$} \\
\hline No & $91(34.7)$ & $60(41.4)$ & $31(26.5)$ & \\
\hline \multicolumn{5}{|l|}{ Sleep disturbance } \\
\hline Yes & $166(63.4)$ & $117(80.7)$ & $79(67.5)$ & \multirow[t]{2}{*}{$0.02^{*}$} \\
\hline No & 96 (36.6) & $28(19.3)$ & $38(32.5)$ & \\
\hline \multicolumn{5}{|l|}{ Clinical characteristics } \\
\hline \multicolumn{5}{|l|}{ CKD stage } \\
\hline Stage I, II, or III & $104(39.7)$ & 49 (33.8) & $55(47.0)$ & $0.03^{*}$ \\
\hline Stage IV or V & $158(60.3)$ & $96(66.2)$ & $62(53.0)$ & \\
\hline Other chronic diseases & & & & \\
\hline$\leq 1$ & $90(34.4)$ & $42(29.0)$ & $48(41.0)$ & $0.04^{*}$ \\
\hline$\geq 2$ & $172(65.6)$ & $103(71.0)$ & $69(59.0)$ & \\
\hline
\end{tabular}


Table 1 Demographic and clinical characteristics of all CKD patients with low and high AOD (Continued)

\begin{tabular}{|c|c|c|c|c|}
\hline Hemoglobin (g/dL) & $11.12 \pm 2.04$ & $10.86 \pm 2.11$ & $11.43 \pm 1.89$ & $0.02^{*}$ \\
\hline Serum creatine $(\mathrm{mg} / \mathrm{dL})$ & $4.97 \pm 1.58$ & $5.13 \pm 5.76$ & $4.77 \pm 5.97$ & 0.62 \\
\hline Serum albumin (g/dL) & $3.51 \pm 0.51$ & $3.46 \pm 0.57$ & $3.57 \pm 0.41$ & 0.07 \\
\hline
\end{tabular}

Abbreviation: AOD, Acceptance of disability

Data is presented as mean \pm SD or $n(\%)$

${ }^{\star}$ US $\$ 1=30.23$ NTD at the study time period

${ }^{*} p<0.05$

infection and mortality [24]. Therefore, the implementation of standardized psychosocial assessment and of patient care procedures, in routine care, may help early referral of CKD patients for further therapeutic interventions that could delay the progression of disease.

The findings of our study revealed that CKD patients with stage IV and above had a higher risk of poor clinical outcomes, and this is compatible with a large population-based study showing that late-stage CKD was a significant risk factor for mortality among renal failure patients in Taiwan [25]. It could be argued that patients with advanced stage of kidney failure typically experience higher predisposition to cardiovascular diseases or renal-related co-morbidities, and consequently are at an elevated risk of death [26]. Additionally, the present study showed that a one-unit increase in hemoglobin was associated with a $19 \%$ decrease in the risk for the composite end-point, and this is compatible with findings of a previous study [27]. Patients with decreased levels of hemoglobin are more prone to anemia, which can evoke left ventricular hypertrophy and cardiovascular mortality [28]. Therefore, subnormal hemoglobin levels in CKD patients should be recognized and managed at earlier stages of disease progression.

\section{Strengths and limitations}

In contrast to the available data on the influence of psychological responses on clinical outcomes in renal disease patients who receive dialysis treatment, the findings from this 3-year prospective cohort study focused on CKD subjects who did not yet undergone dialysis. Our findings provide strong support for the importance of psychological factors, such as disability acceptance, in affecting clinical outcomes, thus supporting usefulness of

Table 2 Effect of AOD on risk of death or progression to dialysis in CKD patients

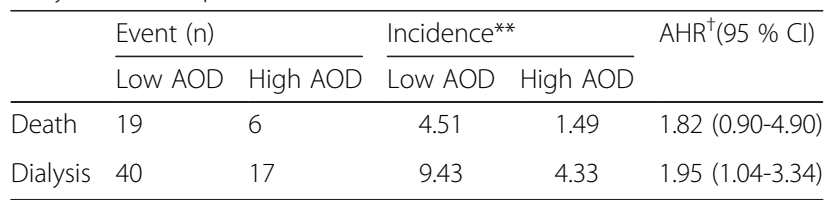

Abbreviations: AOD, Acceptance of disability; AHR, adjusted hazard ratios **Incidence is presented as cases per 1000 person-months ${ }^{+}$Adjusted for the educational level, exercise habit, CKD stage, sleep disturbance, chronic disease, and hemoglobin level therapeutic regimens geared at promoting psychosocial adjustment to CKD. Several limitations, nevertheless, should be considered when interpreting the results of the present study. First, all participants were drawn from a single hospital in southern Taiwan, so the results might not be generalizable to other CKD subjects, especially those from non-Chinese populations. However, previous research has also often been limited by such factors as subjects' ethnicity, geographical location, nationality and the nature of the medical data available, suggesting that this limitation is not unique to our study. Second, in this study, we only assessed AOD at the beginning of the follow-up time period. It is likely that AOD scores could change over time. However, failure to consider the effect of time-varying predictors on end-points would tend to underestimate, rather than overestimate, an association

Table 3 Multivariable analysis of factors associated with the composite endpoint of death and progression to dialysis

\begin{tabular}{|c|c|c|}
\hline Variables & CHR $(95 \% \mathrm{Cl})$ & $\mathrm{AHR}^{\dagger}(95 \% \mathrm{Cl})$ \\
\hline \multicolumn{3}{|l|}{ AOD } \\
\hline Low & $2.34(1.51-3.49)^{*}$ & $1.89(1.18-3.20)^{*}$ \\
\hline High & - & - \\
\hline \multicolumn{3}{|l|}{ Educational level } \\
\hline Low & $0.98(0.49-1.38)$ & $1.01(0.58-1.71)$ \\
\hline High & - & - \\
\hline \multicolumn{3}{|c|}{ Other chronic diseases } \\
\hline$\leq 1$ & $1.34(0.82-2.17)$ & $1.31(0.81-2.13)$ \\
\hline$\geq 2$ & - & - \\
\hline \multicolumn{3}{|l|}{ Regular exercise } \\
\hline No & $0.89(0.41-1.57)$ & $0.97(0.50-1.44)$ \\
\hline Yes & - & - \\
\hline \multicolumn{3}{|l|}{ Sleep disturbance } \\
\hline Yes & $1.17(0.74-2.84)$ & $1.04(0.94-2.98)$ \\
\hline No & - & - \\
\hline \multicolumn{3}{|l|}{ CKD stage } \\
\hline Stage IV or V & $2.26(1.70-5.36)^{*}$ & $1.82(1.21-4.21)^{*}$ \\
\hline Stage I, II, or III & - & - \\
\hline Hemoglobin ( $\mathrm{g} / \mathrm{dL}$ ) & $0.78(0.63-0.88)^{*}$ & $0.81(0.70-0.92)^{*}$ \\
\hline
\end{tabular}

Abbreviations: AOD, Acceptance of disability; CHR, crude hazard ratios; AHR, adjusted hazard ratios

${ }^{\dagger}$ Adjusted for the other variables in this table ${ }^{*} p<0.05$ 
[29], so AOD is more likely to increase rather than decrease over time [14]. Therefore, this potential concern is not likely to influence the conclusions of our study. Third, we assessed sleep disturbance using a single-item question, so the psychometric soundness of such an assessment may be suspect and thus must be interpreted cautiously.

\section{Implications}

The findings of this study have important implications for future research and clinical practice. Given that our data were drawn from a single hospital may restrict the generalizability of these findings. Accordingly, it is recommended, that larger community-based studies, with a comprehensive battery of psychological and clinical measures, be employed to identify undiagnosed patients in order to validate our findings. Furthermore, longitudinal measurement of disability acceptance should be implemented to verify if the present findings are consistent across the process of psychological adaptation to CKD. As far as clinical practice is concerned, the findings of this study indicate that AOD level could serve as an independent risk factor for poor clinical outcomes, suggesting that the AODS-R scale could be judiciously applied when assessing reactions towards life with a chronic illness, such as CKD. Finally, the present findings, especially if supported by future research, also point to the importance of routinely observing patients for negative psychological responses, and, if feasible, instituting appropriate interventions for routine clinical practice.

\section{Conclusion}

We found that low AOD was independently associated with an increased risk of poor clinical outcomes. In addition, patients with CKD stage IV and above and those with low hemoglobin levels also had an elevated risk of poor clinical outcomes. These findings, together with previous results, provide further support that poor psychosocial adaptation, as assessed by low levels of disability acceptance, may have an imperative role in CKD prognosis. Therefore, it may be tentatively recommended that routine psychological screening, through the use of AOD at diagnosis of CKD, followed by monitoring and timely management of its progression, could be beneficial to patients with CKD.

\section{Abbreviations}

AHR: Adjusted hazard ratios; AOD: Acceptance of disability; AODS-R: Acceptance of Disability Scale-Revised; CHR: Crude hazard ratios; Cl: Confidence interval; CKD: chronic kidney disease; CRP: C-reactive protein; GFR: glomerular filtration rate; HPA: Hypothalamic-pituitary-adrenal; IL-6: Interleukin 6; PMs: Personmonths; SD: Standard deviation; TDQ: Taiwan Depression Questionnaire; TNF-a: Tumor necrosis factor-a.
Competing interests

No conflict of interest has been declared by the authors.

\section{Authors' contributions}

$\mathrm{HHC}$ and MLY was responsible for data collection and participated in providing comments on the manuscript drafts. HRG and HL contributed to the interpretation of data and providing comments on the final draft of the manuscript. TYT was responsible for the study conception, design, data analysis, and drafting of the work. All authors read and approved the final manuscript.

\section{Acknowledgements}

This study was supported by Grant DTCRD102(2)-I-08 from the Buddhist Dalin Tzu Chi Hospital. HHC, HRG and HL contributed equally to this work.

\section{Author details}

'Department of Nursing, Dalin Tzuchi Hospital, The Buddhist Tzuchi Medical Foundation, Chiayi, Taiwan. ${ }^{2}$ College of Nursing, Chang Gung University of Science and Technology, Chiayi, Taiwan. ${ }^{3}$ Rehabilitation Counseling Program, Portland State University, Oregon, USA. ${ }^{4}$ Department of Environmental and Occupational Health, College of Medicine, National Cheng Kung University, Tainan, Taiwan. ${ }^{5}$ Department of Occupational and Environmental Medicine, National Cheng Kung University Hospital, Tainan, Taiwan. ${ }^{6}$ Department of Medical Research, Dalin Tzuchi Hospital, The Buddhist Tzuchi Medical Foundation, No. 2, Minsheng Rd., Dalin Township, Chiayi County 62247ROC, Taiwan. ${ }^{7}$ Department of Nursing, Tzu Chi University of Science and

Technology, Hualien, Taiwan.

Received: 20 April 2015 Accepted: 25 November 2015

Published online: 05 December 2015

\section{References}

1. El Nahas AM, Bello AK. Chronic kidney disease: the global challenge. Lancet. 2005;365:331-40.

2. Lysaght MJ. Maintenance dialysis population dynamics: current trends and long-term implications. J Am Soc Nephrol. 2002;13:S37-40.

3. National Health Insurance Administration. Statistics and Surveys. 2012. http://www.nhi.gov.tw/English/webdata/webdata.aspx?menu=11\&menu_ id=296\&WD_ID=296.\&webdata_id=4456.

4. Wen CP, Cheng TY, Tsai MK, Chang YC, Chan HT, Tsai SP, et al. All-cause mortality attributable to chronic kidney disease: a prospective cohort study based on 462293 adults in Taiwan. Lancet. 2008;371:2173-82.

5. Palmer SC, Vecchio M, Craig JC, Tonelli M, Johnson DW, Nicolucci A, et al. Association between depression and death in people with CKD: a metaanalysis of cohort studies. Am J Kidney Dis. 2013;62:493-505.

6. Feng L, Yap KB, Ng TP. Depressive symptoms in older adults with chronic kidney disease: mortality, quality of life outcomes, and correlates. Am J Geriatr Psychiatry. 2013;21:570-9.

7. Livneh H, Antonak RF. Psychosocial adaptation to chronic illness and disability: a primer for counselors. J Counsel Dev. 2005;83:12-20.

8. Attawong T, Kovindha A. The influencing factors of acceptance of disability in spinal cord injuries patients. Nepal J Neurosci. 2005;2:67-70.

9. Groomes DAG, Linkowski DC. Examining the structure of the revised acceptance disability scale. J Rehabil. 2007;73:3-9.

10. Li L, Moore D. Acceptance of disability and its correlates. J Soc Psychol. 1998;138:13-25.

11. Linkowski D. A scale to measure acceptance of disability. Rehabil Couns Bull. 1971;14:236-44.

12. Berglund BA, Mattiasson AC, Nordstrom G. Acceptance of disability and sense of coherence in individuals with Ehlers-Danlos syndrome. J Clin Nurs. 2003; 12:770-7.

13. Richardson A, Adner N, Nordstrom G. Persons with insulin-dependent diabetes mellitus: acceptance and coping ability. J Adv Nurs. 2001;33:758-63.

14. Chiu SY, Livneh H, Tsao LL, Tsai TY. Acceptance of disability and its predictors among stroke patients in Taiwan. BMC Neurol. 2013;13:175.

15. Chen RK, Crewe NM. Life satisfaction among people with progressive disabilities. J Rehabil. 2009;75:50-8.

16. Chiang HH, Lai NS, Livneh H, Yeh ML, Tsai TY. Validity and reliability of Chinese version of acceptance of disability scale-revised. Tzuchi Nurs $J$. 2013;12:66-76 
17. Hedayati SS, Minhajuddin AT, Afshar M, Toto RD, Trivedi MH, Rush AJ. Association between major depressive episodes in patients with chronic kidney disease and initiation of dialysis, hospitalization, or death. JAMA. 2010;303:1946-53.

18. Fischer MJ, Kimmel PL, Greene T, Gassman JJ, Wang X, Brooks DH, et al. Elevated depressive affect is associated with adverse cardiovascular outcomes among African Americans with chronic kidney disease. Kidney Int. 2011;80:670-8

19. Lacson E, Li N-C, Guerra-Dean S, Lazarus M, Hakim R, Finkelstein FO. Depressive symptoms associate with high mortality risk and dialysis withdrawal in incident hemodialysis patients. Nephrol Dial Transplant. 2012;27:2921-8.

20. Cohen SD, Norris L, Acquaviva K, Peterson RA, Kimmel PL. Screening, diagnosis, and treatment of depression in patients with end-stage renal disease. Clin J Am Soc Nephrol. 2007;2:1332-42.

21. Kimmel PL. Depression in patients with chronic renal disease: what we know and what we need to know. J Psychosom Res. 2002;53:951-6.

22. Simic Ogrizovic S, Jovanovic D, Dopsaj V, Radovic M, Sumarac Z, Bogavac $\mathrm{SN}$, et al. Could depression be a new branch of MIA syndrome? Clin Nephrol. 2009;71:164-72.

23. Dowlati Y, Herrmann N, Swardfager W, Liu H, Sham L, Reim EK, et al. A meta-analysis of cytokines in major depression. Biol Psychiatry. 2010;67:446-57.

24. Dalrymple LS, Go AS. Epidemiology of acute infections among patients with chronic kidney disease. Clin J Am Soc Nephrol. 2008;3:1487-93.

25. Hwang SJ, Lin MY, Chen HC, Hwang SC, Yang WC, Hsu CC, et al. Increased risk of mortality in the elderly population with late-stage chronic kidney disease: a cohort study in Taiwan. Nephrol Dial Transplant. 2008;23:3192-8.

26. Chiu YL, Chien KL, Lin SL, Chen YM, Tsai TJ, Wu KD. Outcomes of stage 3-5 chronic kidney disease before end-stage renal disease at a single center in Taiwan. Nephron Clin Pract. 2008;109:C109-18.

27. Yang W, Israni RK, Brunelli SM, Joffe MM, Fishbane S, Feldman HI. Hemoglobin variability and mortality in ESRD. J Am Soc Nephrol. 2007;18:3164-70

28. Ofsthun N, Labrecque J, Lacson E, Keen M, Lazarus JM. The effects of higher hemoglobin levels on mortality and hospitalization in hemodialysis patients. Kidney Int. 2003;63:1908-14.

29. van Walraven C, Davis D, Forster AJ, Wells GA. Time-dependent bias was common in survival analyses published in leading clinical journals. J Clin Epidemiol. 2004;57:672-82.

\section{Submit your next manuscript to BioMed Central and we will help you at every step:}

- We accept pre-submission inquiries

- Our selector tool helps you to find the most relevant journal

- We provide round the clock customer support

- Convenient online submission

- Thorough peer review

- Inclusion in PubMed and all major indexing services

- Maximum visibility for your research 